\title{
Towards Requirement Change Management for Global Software Development using Case Base Reasoning
}

\author{
SADIA ALI*, NAILA IQBAL* AND YASER HAFEEZ* \\ RECEIVED ON 20.10.2017 ACCEPTED ON 12.02.2018
}

\begin{abstract}
Software development globally an important business nowadays and also there are different issues related to software development process especially in change management process when there is change in requirement due to lack of communication, coordination and expert knowledge not properly manage. The aim of this paper is to provide and proposed framework to manage RCM (Requirement Change Management) using CBR (Cased Based Reasoning) technique effectively and intelligently. CBR to solve change in requirement on the base of previous knowledge and experience evaluated the proposed framework through experimental study. The experimental study depicted that proposed framework improved requirement change management in GSD (Globally Software Development) instead of other methods for change management of requirements in globally dispersed environment effectively.
\end{abstract}

Key Words: Global Software Development, Challenges, Requirement Change Management, Requirement Change Management in Global Software Development, Artificial Intelligent Techniques in Requirement Change Management, AI Techniques.

\section{INTRODUCTION}

$\mathrm{S}$ oftware Client Development is more popular among software development organizations globally due to growth in information and computer technologies. The GSD increased efficiency and reduce cost, increased quality of product and accuracy due to high expertise from different locations [1-3].

In GSD environment, both the client and development team are geographically disbursed as shown in Fig. 1. Hence there are different processes involved in the lifecycle of software development and to complete the lifecycle client and development team both have to communicate and collaborate with each other [1-4].

The main challenges in GSD are communication, coordination and control which impact on the all activities of requirement engineering in GSDas describe in Table 1 [1-4]. The reasons of this issue are language, different time zone and cultures, lack of face to face conversation, communication medium, Lack of awareness, trust and sharing. While there is the lack of central database to store and manage the requirement [1-4]. 
In software development lifecycle among all processes requirement engineering process play an important role and success or failure depends on the RCM activities $[4,5,8]$.

The process of RCM is interpreting, managing, analysing, monitoring, tracing and verifying changes in requirements as shown in Fig. 2 [3,16,19,26,]. In RCM process the client submit the request then change control board make impact analysis and change manager on the base of analysis make decision about the change. After the acceptance of change developer develops, implement and update documents then tester verify the changes.To fulfil the changing needs of stakeholders/customers necessary for

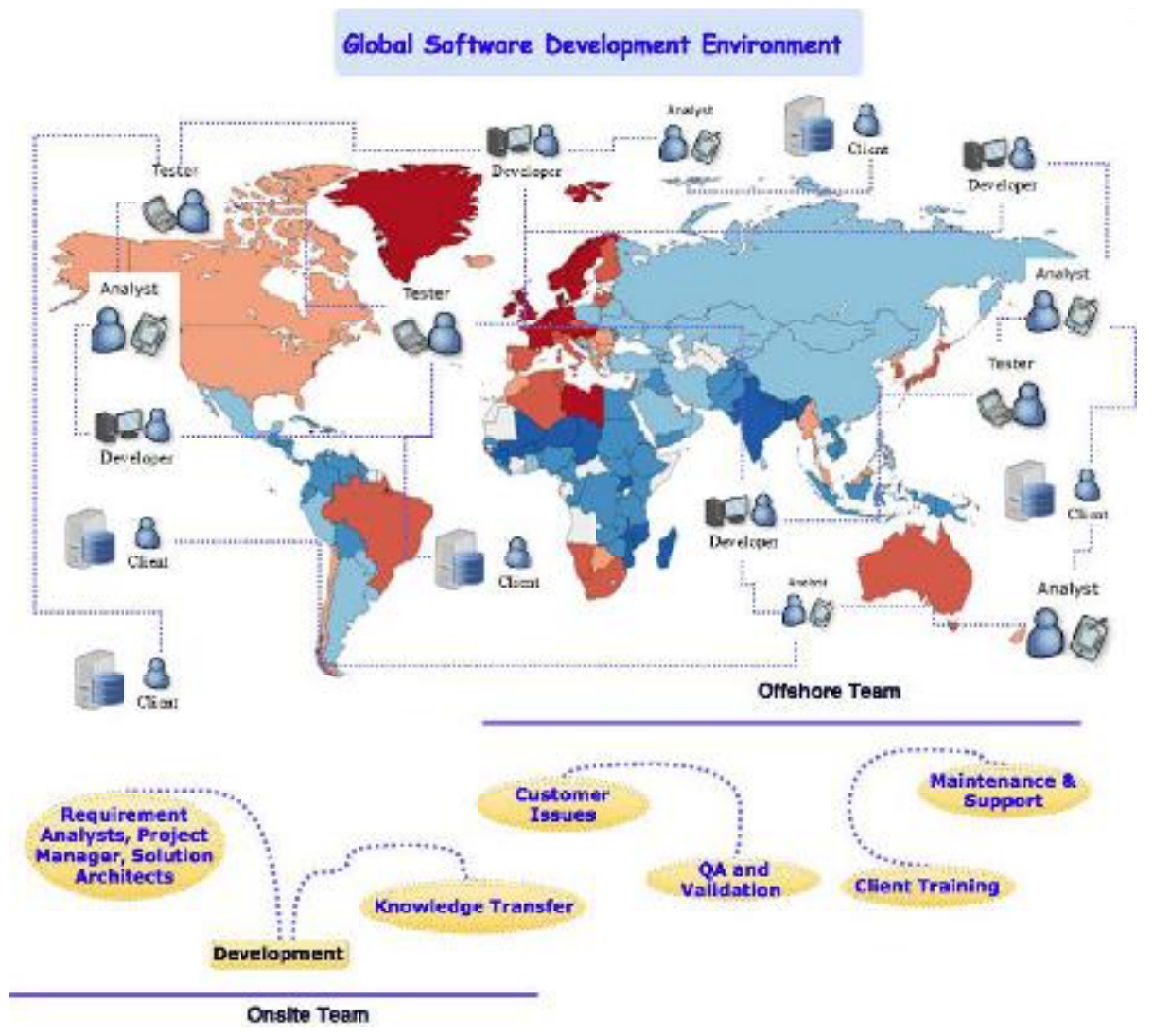

FIG. 1. GLOBAL SOFTWARE DEVELOPMENT [1,4]

TABLE 1. GSD CHALLENGES [1-4]

\begin{tabular}{|c|c|}
\hline GSD Challenges & Description \\
\hline Communication & $\begin{array}{c}\text { Involves for information exchange and causes; } \\
\text { Misunderstandings, delays, less informal exchange, different languages, different domain knowledge. }\end{array}$ \\
\hline Coordination & $\begin{array}{c}\text { Involves for groups activities and artifacts so they contribute to the overall objective, build a team and task awareness. } \\
\text { Control }\end{array}$ \\
$\begin{array}{c}\text { Involves for goals and policies and artefacts management } \\
\text { Correct perception } \\
\text { Accurate status information }\end{array}$ \\
Different uniform tools and techniques use for process. \\
\hline
\end{tabular}

Mehran University Research Journal of Engineering \& Technology, Volume 37, No. 3, July, 2018 [p-ISSN: 0254-7821, e-ISSN: 2413-7219] 
the success of software applications, as a result, the requirements changing becomes challenging $[3,5,7,8,10]$.

So, the main issue in GSD is to manage the change in the requirements completely and effectively according to client need without disturbing other requirements and budget. Therefore, need of framework to solve the problem in better and intelligent way.

The researchers and developers work to improve the software development in different way to reduce the failure rate and software cost especially in GSD environment. They integrated the AI (Artificial Intelligence) techniques for software development activities like risk assessment, reusability and testing $[6,7]$.

AI techniques which base on expert and domain knowledge, previous experience and past projects such as knowledge based system, neural networks, fuzzy logic, machine learning and case base reasoning to improve the software quality [7]. So, in this paper AI technique integrated with traditional method of software engineering to solve the RCM issues in GSD $[8,9]$.

Therefore, there's need of technique which provide the central database with expert and domain knowledge, experience, solutions and projects for the RCM with better communication and coordination.

In GSD environment especially, where team and client are not on the same place, different languages and different cultures etc. [10]. All these differences create the problem of communication and coordination especially in RE process which leads to increase in cost, effort and project failure [12-16]. The objectives of the study are to scrutinize the GSD challenges during RCM process and proposed framework to manage these challenges in an efficient way.

The proposed framework used to manage the RCM in GSD using CBR. CBR is an AI technique base on the

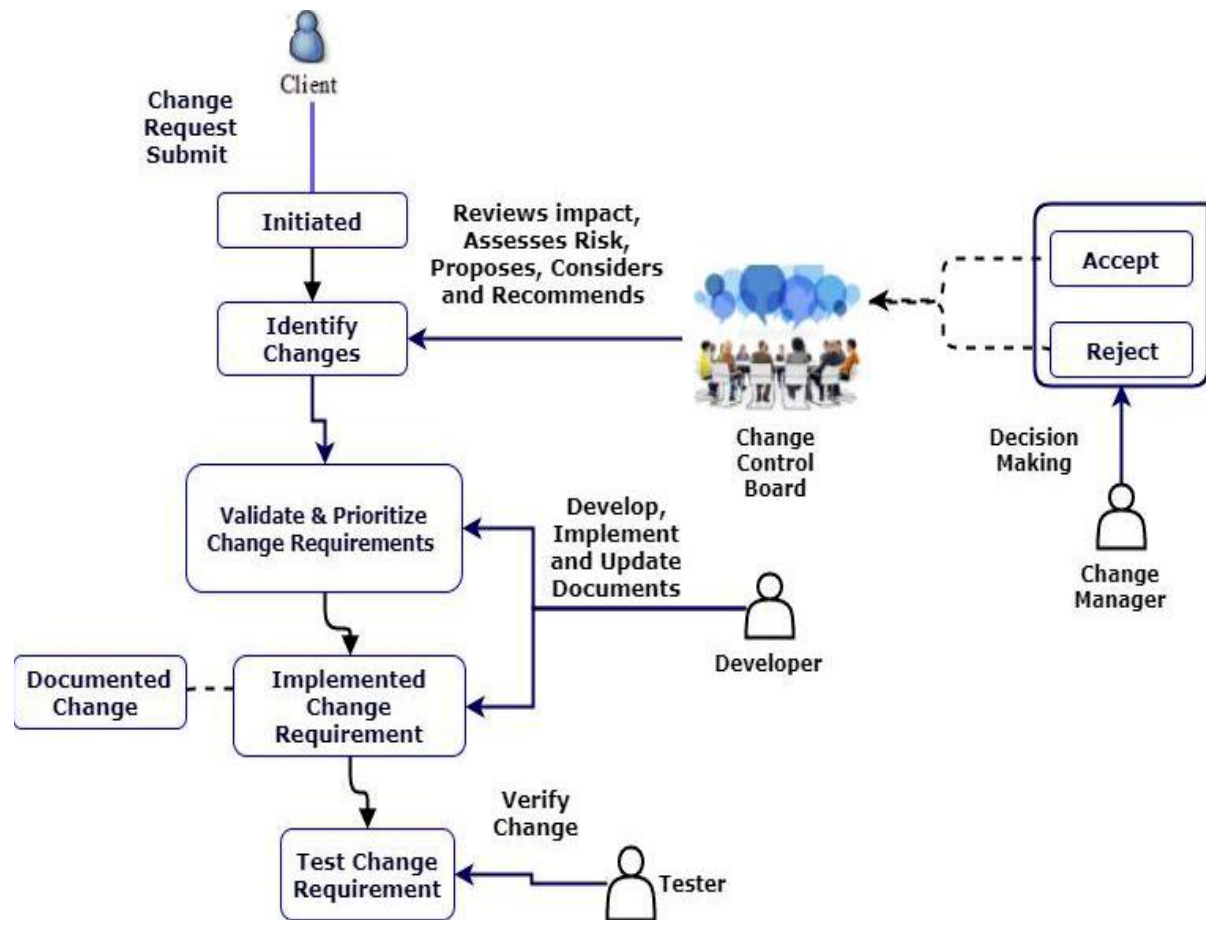

FIG. 2. RCM PROCESS [4,5,8] 
expert knowledge and previous experiences in better an intelligent way with less time, effort and cost $[6,17]$.

CBR is seems to be an attractive approach because it offers transparency and continuous improvement as experience is gained $[6,22]$. The CBR work on the concept of reuse the solution of previous cases like new case and store the cases in the central database for future use [17].

So, this paper introduces an approach for GSD to manage change in requirement using CBR technique to fill the gap between the industry and research in distributed development and then implemented the approach in the industrial dataset. The main purpose of proposed framework to mitigate RCM challenges facing distributed environment effectively using AI techniques. To control and manage requirements changes we suggest new framework to represent the processes of adding, updating and deleting requirements during development process in GSD.

In related work we critically analysed the existing studies to identify the methods of RCM in GSD and limitations of these methods for change management in global environment. Some of these existing studies [16] applied the agile methods in GSD to reduced costs, higher efficiency and better customization, by managing requirements change using feature model called a features tree associated with software tool. The approach evaluated in real environments by software development professionals using an exhaustive set of criteria, and the results are promising. The approach used medium size database which only used for the change document management and not maintain the previous cases for reusability.

Shalinka and Lai [10] presented a systematic review of research in RCM and highlighted four important elements of manage requirement change i.e. causes, processes, techniques of RCM and decisions making in RCM. So, this study has provided information about the current state-of-the-art techniques and practices for RCM and guideline for software practitioners who will be in a position of making better decisions on RCM activities. There is no guideline for the management of requirements change.

Ahmed and Amir [17] proposed a framework in which he discuss the standardize and the positive result in the global software development environment. This framework may be useful to make better comprehension and behavior involved in GSD. RCM is inappropriate to handle because of coordination and communication barriers. But proposed framework is used for only one organization and not for large scale organizations.

Waqar [18] performed different case studies in which he explored the encounters tackled by practitioners and the helpful as well as delaying role of CTs (Collaborative Technologies) in carrying out RCM activities RCM poses continuous challenges to globally distributed software projects. Gathering data from interview question may take a lot of time and resources.

Habiba et. al. [19] suggested the framework for the change which will be managed the requirements, that will come from different phases of Software development which effect is to minimize uncertainty of failure by handling fluctuating requirements but there is still need to used GSD instead of RCM.

Rafiq et. al. [14] review the literature and has criticized that issue like communication, face to face communications, cultural differences and coordination faces during OSDO organizations. These results reveal that focusing on these practices can help vendor organizations to strengthen their relationships with client organizations in OSDO. But this paper present only one component. 
Abdulaziz and Hammed [11] proposed a FreGsd framework with regards to developing and handling requirements discourse the communication and collaboration among different sites. The framework helps organizations to develop in distributed locations to evade common mistakes that lead to recurrent failure and apply best methods to maximize the efficiency and to develop and manage requirements in GSD environments.

Nasir and Zulfiqar [20] perform Systematic literature review and proposed framework for RCM in global development environment. The objective of proposed framework to control the requirement change specifically in GSD environment in apposite manner; framework follows the required processes of requirement change and reduces the concerns of GSD. The RCM process becomes more complicated in dispersed environment due to the lack of communication and teamwork among globally dispersed stakeholders.

Saiqa et. al. [9] perform systematic literature review and identified the issues during RCM in global software development environment. During SLR different issues has been recognized and conclude that on the certain parameters, not for all the parameters cloud computing provide a satisfaction result for change management.

Huma et. al. [12] performed systematic literature review and diagnoses the hindrance produce by RCM during the process of GSD. This research produces base for the computer engineers to resolve risk and hindrance related to RCM in new ways.

Existing studies manage RCM in the distributed environment but still there are some challenges of the existing GSD methods during RCM which we incorporatedin our proposed framework such as Communication, Coordination, Control, Knowledge Management, repository management etc. $[2,5,9,26]$.
From the existing studies we analysed that different methods adopted for the RCM but less focused in the globally disseminated environment due to deficiency of communication, coordination and control because need different methods and tools needed to solve these issues. Also there is no proper management of old RCM problem solutions. So, there is need of a method for problem solving and learning tomanage requirements change using the previous cases and experts' knowledge by sharing central repository like cloud computing by the software organization to develop project in globally dispersed team and clients.

The paper organized as material and methods, results and discussion, conclusion and future works in section II, III and IV respectively.

\section{MATERIALS AND METHOD}

We first critically analyzed the problems from the existing literature during RCM in GSD and on the base of problems proposed framework to overcome the identified issues.

\subsection{Proposed Framework}

The proposed framework use to manage the RCM in GSD using CBR. CBR is an AI technique base on the expert knowledge and previous experiences in better an intelligent way with less time, effort and cost.

CBR is seems to be an attractive approach because it offers transparency and continuous improvement as experience is gained $[16,21]$. The CBR work on the concept of reuse the solution of previous cases similar to new case and store the cases in the central database for future use.

CBR is the logic based method to solve the problem in four different stages i.e. retrieving, reusing, revising and retaining $[22,16]$. In first stage the most similar case or cases retrieve. Second stage the problem is resolve and we reuse the facts in that case. Third step we review the 
planned solution and finally in last step we retain this experience expected to be useful for future problem solving $[6,17]$.

On this logical concept CBR in cloud used to propose the framework for RCM. In cloud computing each team member and client does not have to install many tools like communication tools and coordination tools for the software development $[1,6,23]$.

Cloud provides all the services and platform to client and development team especially in GSD environment to use any service required on demand basis with no constraint of time and place [1,9]. CBR in cloud for GSD team and clients helps to communicate and manage change in requirement intelligently and beneficial for future change in requirement.

The following steps adopted for the RCM in GSD environment and Fig. 3 depicts the proposed framework.

\subsubsection{Change Request}

New request from the client initiated for the change in requirements and communicated through site of the organization.

\subsubsection{Change Control System}

In the change control system developer team identify and analyse the change from the previous cases relevant to the requirements of recent change in requirements of client. Change control system performs following functions for RCM.

Retrieve Case: In this new request considered as new case and compare with previous similar cases to find similar cases. Similar case select the relevant case and retrieve then adopt case for further process.

Reuse Case: The selected case which similar to previous cases, reuses to fulfil the change in requirement for higher satisfaction of client.

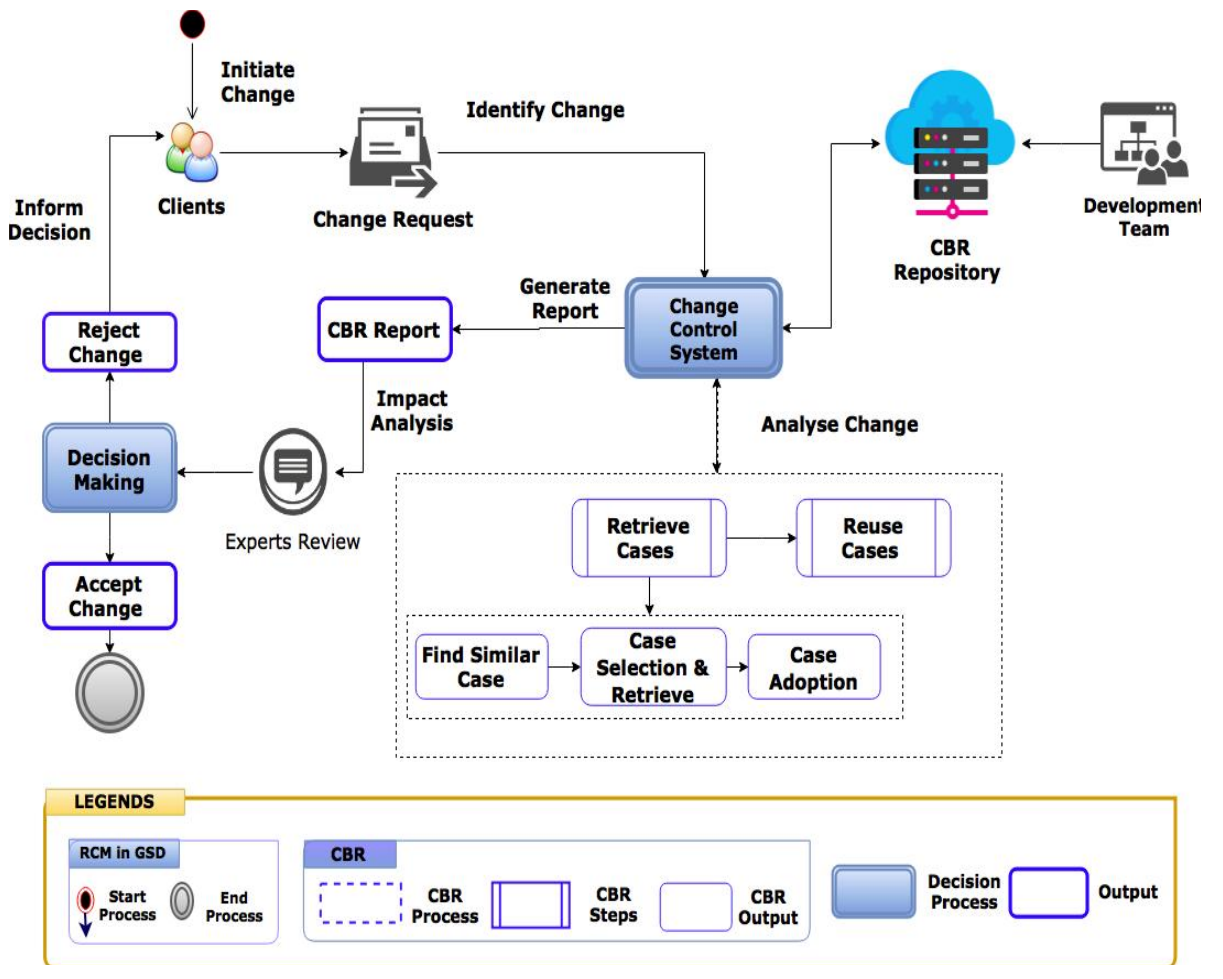

FIG. 3. PROPOSED FRAMEWORK FOR RCM USING CBR

Mehran University Research Journal of Engineering \& Technology, Volume 37, No. 3, July, 2018 [p-ISSN: 0254-7821, e-ISSN: 2413-7219] 
Generate CBR Report: The CBR report generate by the change control system for further implications of requirement change. The domain experts review the report and analyse on the base of previous knowledge for the impact of the requirement change. Also analyse impact on other requirements and cost of the software analysed. If any effect of requirement change on other requirements then control the issue if possible.

Decision Making: If the changes are possible then accept the change on the base of CBR report and implement the change otherwise reject change and communicated to the client.

CBR Repository: For CBR repository we used TFS (Team Foundation Server) repository for cloud computing in GSD. The TFS is the Microsoft product and used for all the phase of software development like planning, gathering, analysing, developing, testing and maintain the projects. TFS is integrated with different tools and applications such as MS Excel, MS Word, Bugzilla, Eclipse, UML, Power AMC etc.

\section{RESULTS AND DISCUSSION}

For evaluation purpose, we perform empirical study to evaluate the performance of the proposed approach using following research hypothesis.

Ho: $\quad$ Whether using our proposed approach reduces the gaps between clients/stakeholders and development team in GSD?

We know that in GSD there are the gaps of communications and coordination between the clients and the stakeholders. So in this research question we analyze that whether our proposed approach improve the communication and coordination between them.

Ho: Whether using our proposed approach reduces the gaps between software development team in GSD?
We also know that software development team also disperses in GSD environment, so there are also issues of communication, coordination and control between the software development team members. Therefore, in this research hypothesis we analyzed that whether our proposed approach improve the communication and coordination between them.

Ho: Whether using our proposed approach improves the RCM in GSD?

In this hypothesis we analyzed that whether RCM process improves using our proposed approach in GSD.

Ho: $\quad$ Is our proposed approach more effective than other existing RCM approach in GSD?

In this research hypothesis considers whether our proposed approach can handle the RCM in GSD better than other existing traditional RCM techniques.

\subsection{Industrial Study}

For the empirical study we select $\mathrm{ABC}$ Technologies Company due to piracy issue company name not disclose and work the in a GSD environment. The Company head office is in Pakistan, but other branches is in Ireland, Australia, Belgium, New Zealand, Qatar, UAE, UK and South Africa. Company employee more than 300 staff globally.

The company work on different domains such as data management solution and electronic patient care reporting, E-Learning, enhance independent living, emergency dispatch, empowering your business process etc. out of different projects we selected one project i.e. Project-A. Project-A is a development of web based and mobile enable solutions for client services and try to provide easy to use the feature with lower cost and greater user satisfaction. 
ABC Technologies Company used different tools and applications for development to overcome the gaps of communication, coordination and control in GSD. As its main goal to achieve user satisfaction and to provide the interactive interface they were agreed to run our proposed model for development.

In this unit, we present the results of the controlled experiment and analyze them relevant to our research hypothesis above.

\subsection{Controlled Experiment}

A controlled experiment was held for executing the proposed framework in order to check whether it is actually able to provide the services which it promises. A group of 24 members of Project-A involved during the execution of the framework to deal with the requirement change in the globally dispersed ABC Technologies company. These 24 members further categories and described in Table 2.

The proposed framework and existing approach are executed completely. Both of them are used for validating requirements of an application that falls into the domain of e-commerce. The reason behind executing both of these techniques for a similar domain is to make comparison between them worthy. A set of change requirements were initiated for Project-A and some of these requirements are new requirement request, modify requirement request and delete requirement request.

TABLE 2. PROJECT-A MEMBERS DETAIL

\begin{tabular}{|c|c|c|}
\hline No. & Project-A Members & No. of Participant \\
\hline 1. & Clients/Stakeholders & 6 \\
\hline 2. & Team Leaders & 4 \\
\hline 3. & Project Managers & 2 \\
\hline 4. & Software Managers & 2 \\
\hline 5. & Change Managers & 2 \\
\hline 6. & Developers & 6 \\
\hline 7. & Quality Assurance Engineers & 4 \\
\hline
\end{tabular}

We divided the members of Project-A into two groups (i.e. Group-1 and Group-2). The Group-1 handle change requirements request with our proposed approach in which used TFS as cloud repository to overcome the communication, coordination and control challenges and $\mathrm{CBR}$ to deal with requirements changes. The Group-2 use traditional approach for the change management.

The ABC Technology company has a full set of Source Control systems in place but to connect with other team members and clients there is need to require logins, firewalls etc. by the use of TFS services as cloud computing in GSD there is no need to require different software installation and firewalls. TFS connect all the globally dispersed team member and client/stakeholders using single interface and run the entire project with no issues. The TFS provides several options for storing, processing and retrieving data in structured, semistructured and unstructured layouts.

\subsection{Proposed Framework Execution}

The Group-1 members were new to the proposed framework, although they have complete knowledge about the requirement change management process but trained the members to make familiar with TFS services and CBR method. All the members were involved during the execution of this newly proposed framework. The framework had provided a very simple and easy to understand TFS services that assist while performing this critical activity of requirements change in globally distributed software development. Using TFS services all the members were connected at single platform there is no need to use different tools and applications for connection, the change requirements request initiate then send to the change manager apply CBR method then change manager generate reportand attached which seen by every member and decision made. 


\subsection{Traditional Existing Approach Execution}

All the members of Group-2 were involved during in the execution of traditional existing approach. The traditional existing approach used different sites with different logins and members not access with all the process of software development cycles. As certain sites do not have overlapping working hours and long meetings are not feasible even if overlapping time exists. Also this traditional existing approach restricts the usage of English language. This may increase cost, effort, time, ambiguity, incompleteness and other issues like language and cultural differences.

After executing both the approaches we analyzed that our proposed work easy to understand, implementable and improve the communication, coordination and control during development between either team members or between team and stakeholders.

In research hypothesis we conjectures that our proposed framework resolve issues of communication, coordination and control between clients/stakeholders and development team in GSD and also between software development team in GSD. The assessment factors from the literature based on the challenges and issues of techniques used in the RCM and GSD technique such as Communication, Coordination, Control, Completeness, Correctness etc. [2,5,8,10]. Also conjecture that our proposed framework outperforms the traditional existing technique. So we conduct interview from the members of both groups to analyze some of the factors on some parameters which shown and describe inTable 3 and Fig. 4. The results depicts that our approach improves the communication, coordination, control and increases the users/ stakeholders satisfaction.

In Table 4 describe the satisfaction level of the team members of both groups (i.e. Group-1 and Group-2) and the members of Group-1 who adopted the proposed framework more satisfied than the members of Group-2 who adopted traditional exiting approach as shown in Fig. 5. And our proposed approaches work in less time than traditional exiting approach.

TABLE 3. REVIEW ANALYSIS

\begin{tabular}{|c|c|c|c|c|c|}
\hline Parameters & $\begin{array}{c}\text { Strongly Agreed } \\
(\%)\end{array}$ & $\begin{array}{c}\text { Agreed } \\
(\%)\end{array}$ & $\begin{array}{c}\text { Neutral } \\
(\%)\end{array}$ & $\begin{array}{c}\text { Disagreed } \\
(\%)\end{array}$ & Strongly Disagreed (\%) \\
\hline Easy To Understand & 30 & 60 & 8 & 2 & 0 \\
\hline Less Complex & 33 & 59 & 5 & 3 & 0 \\
\hline Easy To Implement & 44 & 53 & 2 & 1 & 0 \\
\hline Easy To Use & 25 & 66 & 7 & 2 & 0 \\
\hline Reduce Effort & 36 & 56 & 6 & 2 & 0 \\
\hline Communication & 43 & 54 & 2 & 1 & 0 \\
\hline Coordination & 44 & 53 & 2 & 1 & 0 \\
\hline Control & 54 & 43 & 2 & 1 & 0 \\
\hline Time & 45 & 53 & 1 & 1 & 0 \\
\hline User Satisfaction & 43 & 54 & 2 & 1 & 0 \\
\hline Lack of Visibility of Tasks & 44 & 53 & 2 & 1 & 0 \\
\hline Completeness & 33 & 59 & 5 & 3 & 0 \\
\hline Correctness & 34 & 58 & 5 & 3 & 0 \\
\hline
\end{tabular}

Mehran University Research Journal of Engineering \& Technology, Volume 37, No. 3, July, 2018 [p-ISSN: 0254-7821, e-ISSN: 2413-7219] 
To grasp the significance difference among proposed and existing techniques we performed statistical analysis using SPSS 19 tool. We perform reliability analysis to check the consistency of the results and performed factor analysis to search variation in the unobserved latent variables. The results are consistent that shown in Table 5 the reliability analysis where Cronbach's Alpha is 0.981 while Table 6 depicts the case processing summary. KMO and Bartlett's test show adequacy and variation are 0.71 and 0.000 respectively in Table 7 . Fig. 6 portray the scree plot of the factor analysis of the approaches.
TABLE 5. RELIABILITY STATISTICS

\begin{tabular}{|c|c|c|}
\hline Cronbach's Alpha & $\begin{array}{c}\text { Cronbach's Alpha } \\
\text { Based on } \\
\text { Standardized Items }\end{array}$ & No. of Items \\
\hline 0.981 & 1.000 & 6 \\
\hline
\end{tabular}

TABLE 6. CASE PROCESSING SUMMARY

\begin{tabular}{|c|c|c|c|}
\hline \multicolumn{2}{|c|}{} & $\mathrm{N}$ & $\%$ \\
\hline \multirow{3}{*}{ Cases } & Valid & 2 & 100.0 \\
\cline { 2 - 4 } & Excludeda & 0 & 0.0 \\
\cline { 2 - 4 } & Total & 2 & 100.0 \\
\hline
\end{tabular}

a. Listwise deletion based on all variables in the procedure.

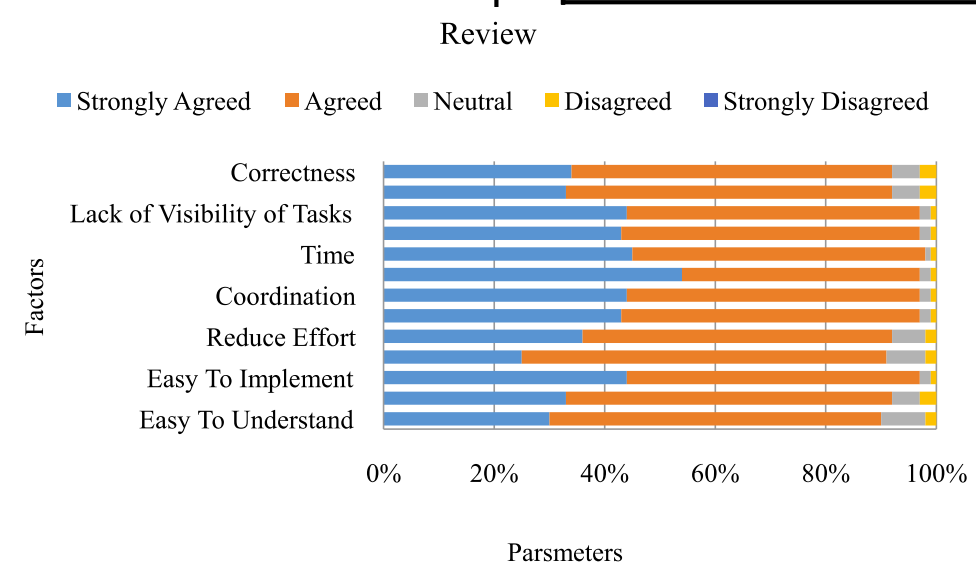

FIG. 4. REVIEW ANALYSIS

Approches Comparison

Without Proposed Framework $\quad$ Proposed Framework

Quality Assurance Engineers

Developers
Change Managers
Team Leaders

Clients/ Stakeholders

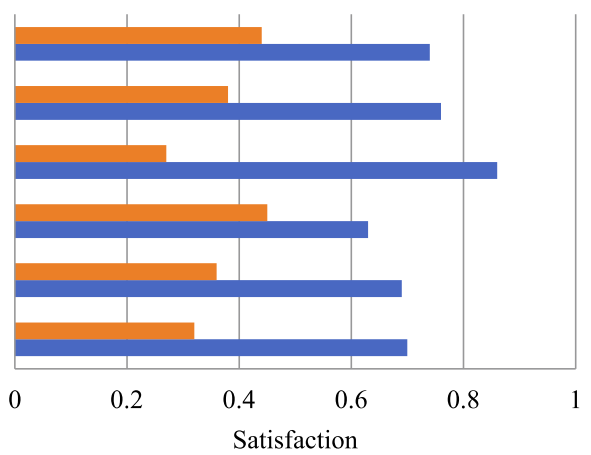

FIG. 5. APPROACHES COMPARISON

TABLE 4. APPROACHES ANALYSIS

\begin{tabular}{|c|c|c|c|c|c|c|}
\hline \multicolumn{9}{|c|}{ PARTICIPANTS } \\
\hline & $\begin{array}{c}\text { Clients/ } \\
\text { Stakeholders }\end{array}$ & $\begin{array}{c}\text { Team } \\
\text { Leaders }\end{array}$ & $\begin{array}{c}\text { Project } \\
\text { Managers }\end{array}$ & $\begin{array}{c}\text { Change } \\
\text { Managers }\end{array}$ & $\begin{array}{c}\text { Developers } \\
\text { Quality Assurance } \\
\text { Engineers }\end{array}$ \\
\hline Proposed Framework & 0.7 & 0.69 & 0.63 & 0.86 & 0.76 & 0.74 \\
\hline Without Existing Approach & 0.32 & 0.36 & 0.45 & 0.27 & 0.38 & 0.44 \\
\hline
\end{tabular}

Mehran University Research Journal of Engineering \& Technology, Volume 37, No. 3, July, 2018 [p-ISSN: 0254-7821, e-ISSN: 2413-7219] 
We used paired sample test to check the significant differences between proposed framework and traditional existing approach. From the paired statistics results as shown in Table 8 we analyzed that proposed framework standard deviation (i.e. 9.571) less than the traditional existing approach (i.e. 11.237) and

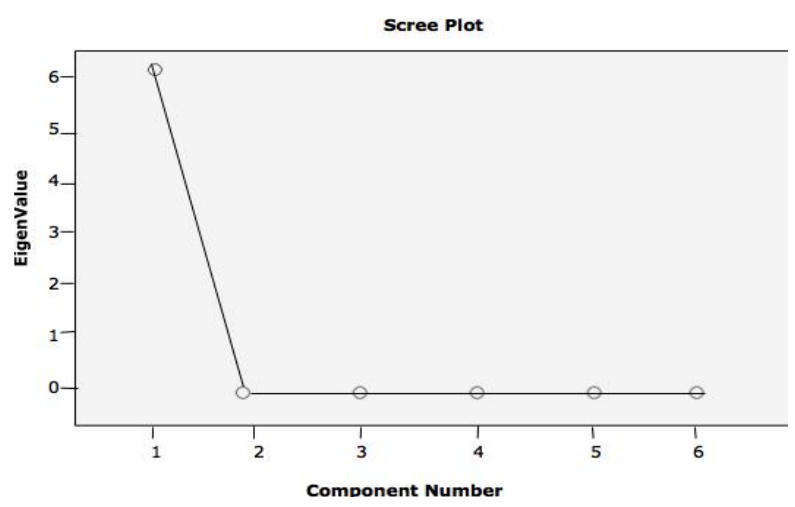

FIG. 6. SCREE PLOT interpreted that proposed framework values less disperses from its mean value, less variable and more reliable than other methods traditional existing approach. Table 9 shows that the the mean difference between groups not equal to zero and p-value is less than the significance level (i.e. $\alpha=0.05$ or $5 \%$ ).

\section{CONCLUSION}

In this paper we have identified the GSD challenges due to requirement change during $\mathrm{RE}$ process and their impacts. The major challenges are the communication, coordination and lack of central database which mainly impacts on RCM activity of requirement engineering process in GSD environment.

TABLE 7. KMO AND BARTLETT'S TEST

\begin{tabular}{|l|c|c|}
\hline \multicolumn{2}{|c|}{ Kaiser-Meyer-Olkin Measure of Sampling Adequacy. } & 0.710 \\
\hline \multirow{3}{*}{ Bartlett's Test of Sphericity } & Approximately Chi-Square & 2.852 \\
\cline { 2 - 3 } & Difference & 1 \\
\cline { 2 - 3 } & Significance & 0.000 \\
\hline
\end{tabular}

TABLE 9. PAIRED SAMPLES STATISTICS

\begin{tabular}{|c|c|c|c|c|c|}
\hline \multicolumn{2}{|c|}{} & Mean & N & Standard Deviation & Standard Eror Mean \\
\hline \multirow{3}{*}{ Pair 1 } & Proposed Framework & 76.40 & 10 & 9.571 & 3.027 \\
\cline { 2 - 6 } & Traditional Existing Approach & 31.60 & 10 & 11.237 & 3.553 \\
\hline
\end{tabular}

TABLE 10. PAIRED SAMPLES TEST

\begin{tabular}{|c|c|c|c|c|c|c|c|c|c|}
\hline & & \multicolumn{5}{|c|}{ Paired Differences } & \multirow{3}{*}{$\mathrm{t}$} & \multirow{3}{*}{ Difference } & \multirow{3}{*}{$\begin{array}{l}\text { Significance } \\
\text { (2-tailed) }\end{array}$} \\
\hline & & \multirow{2}{*}{ Mean } & \multirow{2}{*}{$\begin{array}{l}\text { Standard } \\
\text { Deviation }\end{array}$} & \multirow{2}{*}{$\begin{array}{l}\text { Standard Error } \\
\text { Mean }\end{array}$} & \multicolumn{2}{|c|}{$\begin{array}{c}95 \% \text { Confidence Interval } \\
\text { of the Difference }\end{array}$} & & & \\
\hline & & & & & Lower & Upper & & & \\
\hline Pair 1 & $\begin{array}{c}\text { Proposed } \\
\text { Framework - } \\
\text { Traditional Existing } \\
\text { Approach }\end{array}$ & 44.800 & 18.408 & 5.821 & 31.632 & 57.968 & 7.696 & 9 & 0.000 \\
\hline
\end{tabular}

Mehran University Research Journal of Engineering \& Technology, Volume 37, No. 3, July, 2018 [p-ISSN: 0254-7821, e-ISSN: 2413-7219] 
The proposed framework used CBR technique with cloud i.e. TFS to manage the requirement change in GSD environment with less cost and effort. It makes the communication and coordination reliable and reduces risk due to use of proposed framework for change management. Also our proposed approach outperform with traditional approach both practically and statically. This provides guidelines to GSD developers and researchers. Also helps the software industry to manage change request in global software development.

\section{FUTURE WORK}

In future we will improve trace links due to change in requirements in GSD during requirement engineering process and their impact on requirement engineering activities. This research work will also help cloud environment and federated cloud environment to improve the RCM process.

\section{ACKNOWLEDGEMENT}

Authors would specially thank to our parents who helped a lot in finalizing this research paper. Authors also indebted to Higher Education Commission, Islamabad, Pakistan, for providing facilities and resources to start the work and finally to publish it.

\section{REFERENCES}

[1] Anum, T., Iftikhar, S., and Azam, F.E., “A Novel Cloud Based Data Management Framework for Team and Project Activities in Global Software Development", Journal of Applied Environmental Biological Science, pp. 94-102, 2014.

[2]

Huma, H.K., Mahrin, N., and Chuprat, S., "Environmental Factors Influencing Requirement Engineering in Global Software Development: A Review", International Arab Conference on Information Technology, 2013.
[3] Iqbal, J., Ahmad, R., and Noor, M.A., “A Framework to Improve the Requirements Engineering Process for Software Development Outsourcing”, 22nd Australian Software Engineering Conference, pp. 4-7, Melbourne, 2013.

Klaus, S., "Challenges and Solutions in Global Requirements Engineering: A Literature Survey", Software Quality. Model-Based Approaches for Advanced Software and Systems Engineering, pp. 85-99, Springer, 2014 .

[5] Arif, A.K., Basri, S., and Dominic, P.D.D., "A Propose Framework for Requirement Change Management in Global Software Development”, International Conference on Computer \& Information Science, pp. 944-947, Kuala Lumpeu, 2012.

[6] Hassan, N., Motla, Y.H., Asghar, S., and Khatoon, A., "Effective Usage of AI Technique for Requirement Change Management Practices", IEEE International Conference on Computer Science and Information Technology, pp.121-125, 2013.

[7] Shashank, M.S., and Rath, S.K., "Empirical Assessment of Machine Learning Models for Agile Software Development Effort Estimation Using Story Points", Innovations in Systems and Software Engineering, pp. 1-10, 2017.

[8] Markus, B., Wnuk, K., Regnell, B., and Runeson, P., "Supporting Change Impact Analysis Using a Recommendation System: An Industrial Case Study in a Safety-Critical Context”, IEEE Transactions on Software Engineering, Volume 43, No. 7, pp. 675-700, 2017.

[9] Saiqa, B., Hafeez, Y., Hassan, M.S., Gul, Z., Pervez, H., Ahmed, I., and Mazhar, S., "Requirement Change Management in Global Software Environment Using Cloud Computing”, Journal of Software Engineering and Applications, Volume 7, No. 8, pp. 694, 2014. 
[10] Shalinka, L., and Lai, R., "A Systematic Review of Requirements Change Management", Information and Software Technology, Volume 93, pp.163-185, 2017.

[11] Abdulaziz, A.A., and Hammed, K., "FreGsd: A Framework for Global Software Requirement Engineering”, Journal of Software, Volume 10, No. 10, pp. 1189-1198, 2015.

[12] Huma, H.K., Mahrin, N., and Chuprat, S., "Factors Generating Risks during Requirement Engineering Process in Global Software Development Environment", International Journal of Digital Information and Wireless Communications, Volume 4, No. 1, pp.63-78, 2014.

Petra, B., Smiley, K., and Mohapatra, P., "Global Software Project Management: A Case Study", Software Engineering Approaches for Offshore and Outsourced Development, pp. 64-70, 2010.

Rafiq, A., Khan, S., and Niazi, M., "Communication and Coordination Challenges Mitigation in Offshore Software Development Outsourcing Relationships: Findings from Systematic Literature", Malaysia Special Issue Science International, Volume 26, No. 4, pp. 1425-1429, Lahore, 2014.

Domia, L., Moawad, R., and Kadry, M., "Supporting Tools for Requirements Change Management in Distributed Agile Development”, Future Computing and Informatics Journal, Volume 2, No. 1, pp. 1-9, 2017.

\section{Shi, Y., and Yuechuan, W., "Case Based Reasoning in} Practice: Investigation of the Practical Importance of Case Based Reasoning”, University of Gothenburg Chalmers University of Technology Department of Computer Science and Engineering Göteborg, pp. 1-21, Sweden, 2016.

Ahmed, M., and Amir, H., "Enhancement inthe Effectiveness of Requirement Change Management Model for Global Software Development”, Journal of Science International, Volume 28, No. 2, pp. 1161$1164,2016$.
Waqar, H., "Reflections on Requirements Change Management in Global Software Development: A Multiple Case Study", International Conference on Global Software Engineering Workshops, pp. 77-79, 2016.

Habiba, U., Bokhari, S., and Abaas, M., “A Logic-Based Framework for the Management of Changing Software Requirements", Lecture Notes Software Enginering, Volume 3, No. 4, pp. 275-278, 2015.

Nasir, M., and Zulfiqar, A., "An Improved Framework for Requirement Change Management in Global Software Development", Journal of Software Engineering Applied, Volume 7, No. 8, pp. 779-790, 2014.

Farid, M., and Vadera, S., "Artificial Intelligence Applications for Improved Software Engineering Development: New Prospects: New Prospects", IGI GlobalInformation Science Reference, 2010.

[22] Anum, B., Menzies, T., El-Rawas, O., Kocaguneli, E., and Keung, J.W., “Case-Based Reasoning for Reducing Software Development Effort", Journal of Software Engineering and Applications, Volume 3, No. 11, pp. 1005-1014, 2010.

[23] Victor, H., "Managing Changes Made to Cloud Systems in order to Ensure Data Integrity”, Data Integrity Validation Conference, pp. 1-26, San Diego, CA, 2015.

[24] Anum, T., and Khan, A.A., "Framework Supporting Team and Project Activities in Global Software Development", IEEE International Conference onEmerging Technologies, pp 1-6, 2012.

[25] Khan, A., Shuib, B., and Dominic, P.D.D., "Communication Risks in Global Software Developmentduring RCM: Results from Systematic Literature Review”, IEEE International Conference on Computer and Information Sciences, pp. 1-6, 2014. 
[26] Khan, A., Basri, S., Dominic, P.D.D., and Amin, F.E., "Communication Risks and Best Practices in Global Software Development during Systematic Requirements Change Management: A Literature Review Protocol", Research Journal of Applied Sciences, Engineering and Technology, Volume 6, pp. 3514-3519, 2013.
[27] Muhammad, W.B., Hayat, F., Ehsan, N., Ishaque, A., Ahmed, S., and Mirza, E., “A Methodology to Manage the Changing Requirements of a Software Project", IEEE International Conference on Computer Information Systems and Industrial Management Applications, pp. 319-322, 2010 . 